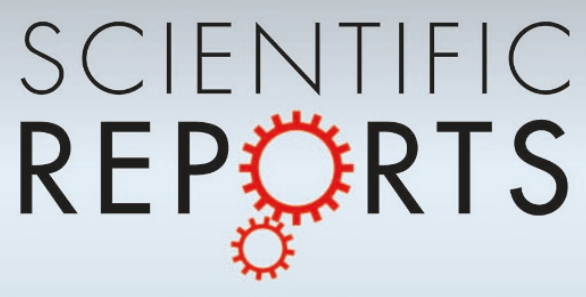

\title{
OPEN Magnetoresistive Emulsion Analyzer
}

\section{SUBJECT AREAS: \\ MICROFLUIDICS}

SENSORS AND PROBES

BIOMEDICAL ENGINEERING

LAB-ON-A-CHIP

Received

25 March 2013

Accepted

6 August 2013

Published

30 August 2013

Correspondence and requests for materials should be addressed to L.B. (larysa.baraban@ nano.tu-dresden.de)

\author{
Gungun Lin 1,2, Larysa Baraban ${ }^{3}$, Luyang Han', Daniil Karnaushenko ${ }^{1,2}$, Denys Makarov', \\ Gianaurelio Cuniberti ${ }^{3,4}$ \& Oliver G. Schmidt ${ }^{1,2}$
}

\begin{abstract}
${ }^{1}$ Institute for Integrative Nanosciences, IFW Dresden, Helmholtzstr. 20, 01069 Dresden, German, ${ }^{2}$ Material Systems for Nanoelectronics, Chemnitz University of Technology, Reichenhainerstr. 70, 09107 Chemnitz, Germany, ${ }^{3}$ Max Bergmann Center of Biomaterials, Dresden University of Technology, Budapesterstr. 27, 01069 Dresden, Germany, ${ }^{4}$ Division of IT Convergence Engineering, POSTECH, Pohang, Korea.
\end{abstract}

We realize a magnetoresistive emulsion analyzer capable of detection, multiparametric analysis and sorting of ferrofluid-containing nanoliter-droplets. The operation of the device in a cytometric mode provides high throughput and quantitative information about the dimensions and magnetic content of the emulsion. Our method offers important complementarity to conventional optical approaches involving ferrofluids, and paves the way to the development of novel compact tools for diagnostics and nanomedicine including drug design and screening.

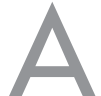

substantial part of nanomedicine focuses on drug design and employing magnetic nanomaterials in combination with multifunctional polymers, lipids or proteins ${ }^{1,2}$. For example, ferrofluid-based liposomes are capable to respond to external stimuli like magnetic fields, which allows the remote control over the chemical species ${ }^{3}$. Ferrofluids are widely used for chemotherapies ${ }^{4}$, in vivo magnetic resonance imaging (MRI) ${ }^{5}$ and as labels in immunological tests, i.e. magnetic enzyme-linked immunosorbent assays (ELISAs) ${ }^{6}$.

High-throughput drug discovery requires a tool which is able to analyze, manipulate, and sort objects, e.g. liposome capsules or emulsions containing known doses of magnetic nanoparticles associated with medications. A promising route relies on the implementation of magnetically-labeled biochemical species in combination with magnetoresistive sensors. In-flow detection based on giant magnetoresistive (GMR) ${ }^{7}$ or tunneling magnetoresistive (TMR) sensors ${ }^{8}$ has been demonstrated recently, representing first steps towards the integration of magnetic sensor elements into fluidic channels.

However, up to date, progress is restrained to the mere sensing and counting of magnetic objects. Advanced and quantitative analysis, which is the main advantage of e.g. optical flow cytometry ${ }^{9}$, has not been explored so far with magnetic sensors. Apart from detection and analysis, sorting of species is invaluable in diagnostic devices. The sorting of biochemical objects has been dominated by conventional fluorescence-activated cell sorting (FACS) techniques ${ }^{10}$.

Here and for the first time, a magnetic emulsion analyzer capable of multiparametric studies and sorting is realized. Ferrofluid emulsion droplets of different size and concentrations of magnetic nanoparticles are produced. The droplet volumes range from $20 \mathrm{nl}$ up to $400 \mathrm{nl}$ - ideally suited for culturing monoclonal cell populations ${ }^{11}$ and monitoring bacteria growth ${ }^{12}$. Multiparametric analysis as well as magnetic sorting of ferrofluid droplets of different size are performed to demonstrate the potential of GMR sensors for magneto-cytometry studies.

\section{Results}

The concept of the magnetoresistive emulsion analyzer capable of multiparametric studies and sorting of ferrofluid droplets is presented in Figure 1a. Water-in-oil emulsion droplets of various sizes with encapsulated ferrofluids are produced in a T-junction geometry. An example of the flow profiles of the two immiscible phases is displayed in Supplementary Figure 6.

The key component in the experimental setup is the GMR-based sensing platform. The sensor is composed of a $\mathrm{Py} / \mathrm{Cu}\left(\mathrm{Py}=\mathrm{Ni}_{81} \mathrm{Fe}_{19}\right)$ layer stack coupled at the 2 nd antiferromagnetic maximum (AFM). The magnetoresistive characteristics (GMR curve and sensitivity) are shown in Figure 1b. A GMR ratio of $11 \%$ and sensitivity of up to $26 \mathrm{~T}^{-1}$ in a magnetic field of $0.9 \mathrm{mT}$ is achieved. The high sensitivity of the GMR sensor and the hysteresis-free sensor response under cycling magnetic field are crucial for the magneto-cytometric detection. The detection is based on a Wheatstone bridge geometry (Supplementary Figure 2). A lock-in amplifier is used to improve the signal-to-noise ratio. Affiliated to the sensing platform is a digital camera to monitor the flowing droplets. Recorded videos and images are correlated with the voltage signals obtained with the GMR sensor. The 


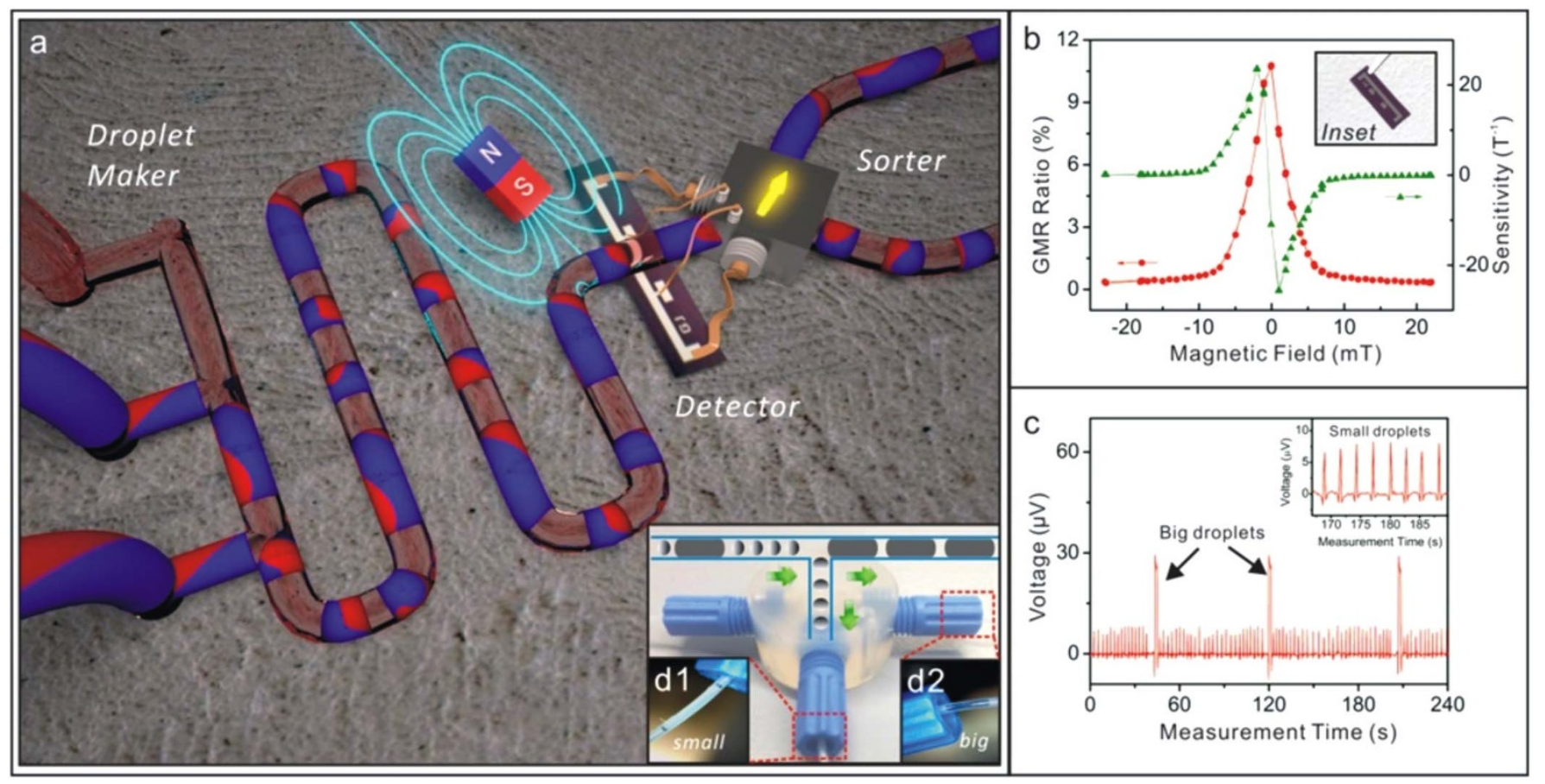

Figure $1 \mid$ (a) Concept of GMR-based droplet analyzer for multiparametric analysis and sorting. (b) Magnetoresistive characterization of GMR sensor. Inset: Photograph of the patterned sensor prepared for measurements in 4-point configuration. (c) Real time detection of a train containing small and large ferrofluid emulsion droplets. Sorting of small (d1) and large (d2) droplets at the T-junction.

water-based ferrofluid (EMG 700, Ferrotec) does not retain remanence without applying an external magnetic field. Therefore, a biasing field is applied to achieve a net magnetization of the ferrofluid, which also serves to operate the sensor in its most sensitive range.

First, we demonstrate the capability of the GMR-based emulsion analyzer to detect and examine ferrofluid droplets of different sizes and concentrations. Each detection event is represented by a peak (Fig. 1c; Supplementary Figure 1a) characterized by its amplitude and the full-width at half maximum (FWHM). These characteristics serve as fingerprints for a ferrofluid droplet, flow conditions and emulsification procedure. For instance, in the case of a fixed sensor-droplet separation, the amplitude is related to the size and concentration of the magnetic material in a droplet, whereas the FWHM contains information about the spread in speed and size of a droplet. Since the following experiments were performed at a fixed flow rate, modifications of the FWHM are mainly attributed to the variation in droplet size.

\section{Discussion}

The multiparametric density plots produced for different volumes of ferrofluid droplets (concentration: $15 \mathrm{mg} / \mathrm{ml}$ ) are summarized in Figure 2a. The broad distribution of the FWHM and amplitude for the smallest droplets (black cloud, Fig. 2a) are consistent with the real time detection results (Supplementary Figure 1a). The corresponding broad standard deviations of the fitted histograms in Figure 2c reflect predominantly the clear polydispersity of droplet sizes, and is to lesser extent ascribed to the inhomogeneity of the ferrofluid concentration between droplets. The polydispersity is partly related to the spontaneous fusion of droplets smaller than the channel dimension. With increasing size (compare red and green clouds), the droplets completely fill the channel cross-section resulting in a more uniform droplet train as reflected by the narrower distribution of the scattered data points around the cloud center (Supplementary Figure 2i). Once the droplet is longer than the width of the magnetic sensor, the sensor voltage change (amplitude) reaches its saturation value and becomes insensitive to the size of the droplets. However, the information about the size of droplets can still be extracted from the FWHM (compare green and blue clouds, Figure 2a). An increase of the FWHM magnitude is observed when the droplet volume increases from $120 \mathrm{nl}$ to $270 \mathrm{nl}$.

Multiparametric analysis performed for a train of droplets (volume: $160 \mathrm{nl}$ ) with different concentrations of ferrofluid is shown in Figure 2b. The clouds of data points for different concentrations naturally fall into the same FWHM region, and the standard deviation of the amplitude and FWHM levels off (Supplementary Figure $2 \mathrm{j}$ ), which implies a relatively high homogeneity of the droplet size and concentration of the ferrofluid for each experiment. The FWHM magnitude within the cloud is mainly determined by the deviation of droplets speed. In contrast, due to the scaling of the voltage change with the magnetic moment, the center of the data point cloud is clearly shifted to higher voltage amplitudes for droplets with larger concentration of magnetic nanoparticles (Figure 2d).

The magnetic droplet analyzer can be extended towards sorting emulsions with respect to a specific property addressed in a multiparametric analysis. The sorting module consists of a split junction and a solenoid 3-way isolation valve installed behind the GMR sensor. For demonstration purposes, we performed sorting of droplets of different sizes, which provide various readouts of the GMR sensor (Figure 1c, Supplementary Movie 1). Small and big droplets were produced in a continuous mineral oil flow ( $Q_{\text {oil }}$ $=200 \mathrm{nl} / \mathrm{s}$ ) using two separated T-junctions (Supplementary Figure 5b) Ferrofluids with a concentration of $75 \mathrm{mg} / \mathrm{ml}$ were used for the sorting experiments. The small droplets (volume: $20 \mathrm{nl}$ ) cause an average voltage change of about $7 \mu \mathrm{V}$, while the big droplets (volume: $500 \mathrm{nl}$ ) provide an average positive voltage change of about $30 \mu \mathrm{V}$. Droplets of different size can be sorted into two separate channels via controlling the isolation valve to be open only when big droplets were passing by the GMR sensor (Supplementary Movie 2). It is foreseeable that various types of emulsion droplets can be sorted with this tool by implementing additional sorting criteria, e.g. dose of the encapsulated magnetic nanoparticles. The demonstration of magnetic-activated sorting 

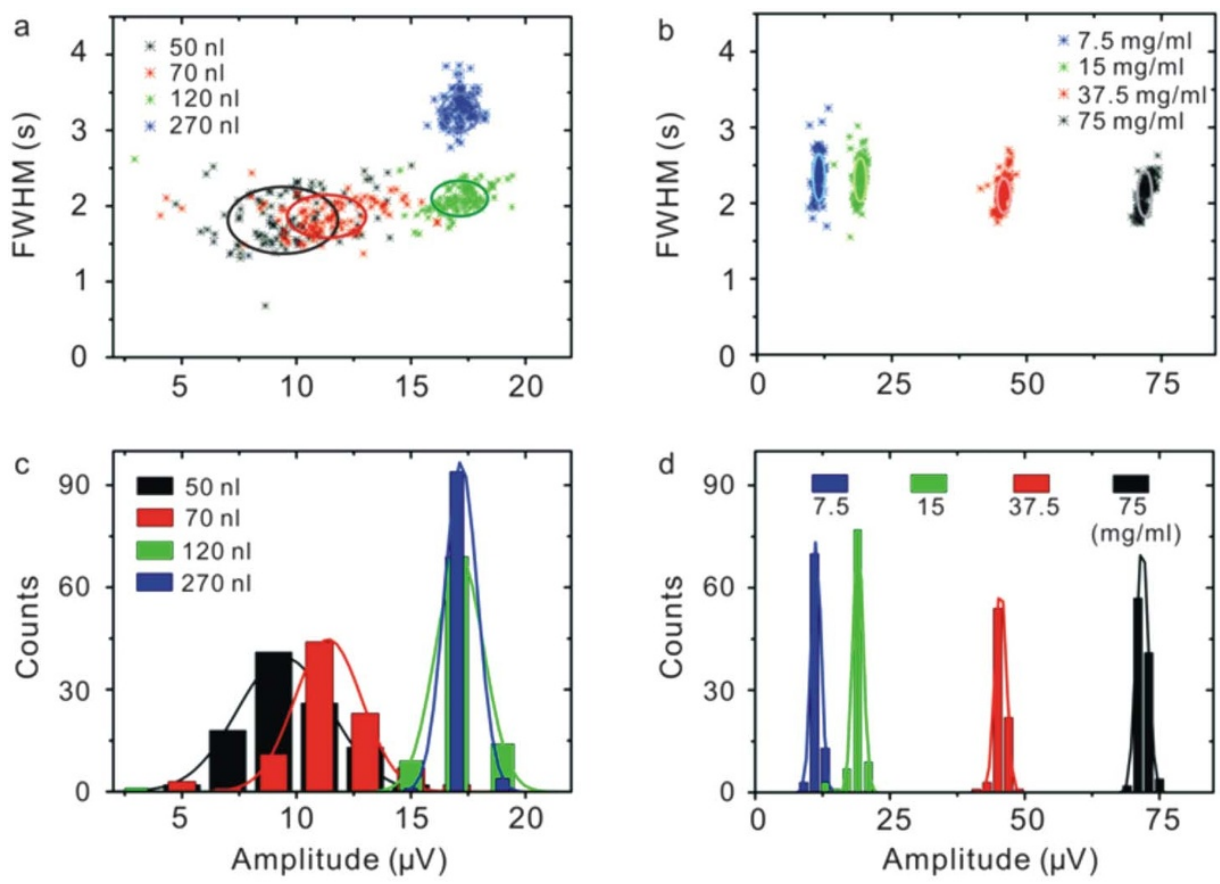

Figure 2 $\mid$ Multiparametric density plots for the detection of magnetic droplets which are produced with (a) different volume of ferrofluid droplets (concentration: $15 \mathrm{mg} / \mathrm{ml}$ ) and (b) different concentrations of ferrofluid in droplets (volume: $160 \mathrm{nl}$ ). The flow rate of mineral oil is $\mathrm{Q}_{\mathrm{oil}}=500 \mathrm{nl} / \mathrm{s}$. Circles are guide to the eyes. Histograms of the voltage amplitude change with (c) different volume of ferrofluid droplets and (d) different concentrations of ferrofluid in droplets. These plots collect measurement data of about 100 droplets.

opens up promising perspectives for drug design and screening, which could be beneficial to the pharmaceutical industry.

\section{Methods}

Droplet generation module. The ferrofluid droplets are formed on a T-junction connector, where aqueous solution of ferrofluid (water-based ferrofluid, Ferrotec EMG Series 700) is dispersed in the mineral oil phase (mineral oil, Sigma-Aldrich M8410). The droplet generator module (Supplementary Fig. 5a) was assembled from several components: the pulse free syringe pumps (neMESYS, Cetoni) for fluid injection; the T-junction for droplet formation; interconnections, i.e. flangeless fittings (standard $1 / 4-28$ thread), syringes (1000 Series, Hamilton, syringe volume: $1.0 \mathrm{ml}$ and $2.5 \mathrm{ml}$ ); flow circuits that were realized with polytetrafluoroethylene (PTFE) tubing with outer diameter (OD) of $0.9 \mathrm{~mm}$ and inner diameter (ID) of $0.4 \mathrm{~mm}$. We used the pulse flow approach to generate droplets of different size for multiparametric analysis. An example of the flow profiles of two immiscible phases are displayed in Supplementary Figure 6. The period of flow pulses is $7 \mathrm{~s}$, while each flow pulse lasts for $500 \mathrm{~ms}$.

Magnetic detection module. The layer stack of the GMR multilayers was: [Py $(1.5 \mathrm{~nm}) / \mathrm{Cu}(2.2 \mathrm{~nm})]_{30} / \mathrm{Py}(1.5 \mathrm{~nm})$, which was prepared by magnetron sputtering technique under high vacuum condition (base pressure: $2.5 \times 10^{-7} \mathrm{mbar}$ ). Ar was employed as the sputter gas, the pressure of which was controlled at $1.6 \times 10^{-3}$ mbar. Before depositing the layer stack, a thermally oxidized silicon wafer with $1000 \mathrm{~nm}$ $\mathrm{SiO}_{\mathrm{x}}$ layer was patterned into rectangular stripe (width: $1 \mathrm{~mm}$, length: $16 \mathrm{~mm}$ ) by standard optical lithography using image reversal technique. The optical lithography was performed in the clean room with standard class 100 conditions. Firstly, the silicon substrate covered with $1 \mu \mathrm{m}$ oxide was spin coated with TI-Prime (MicroChem Corp.) at $4500 \mathrm{rpm}$ for $35 \mathrm{~s}$, followed by a baking at $120^{\circ} \mathrm{C}$ for $3 \mathrm{~min}$. After that, negative photoresist (AZ5214E) was spin coated onto the substrate at $4500 \mathrm{rpm}$ for $35 \mathrm{~s}$, which was subsequently baked at $90^{\circ} \mathrm{C}$ for $4 \mathrm{~min}$. Then the sample was exposed with the stripe pattern using MA56 mask (Karl Süss) aligner for 2 s, and post-baked at $120^{\circ} \mathrm{C}$ for $2 \mathrm{~min}$. Subsequently, the sample was exposed without mask for $30 \mathrm{~s}$ to realize image reversal. At the end, the sample was developed by AZ726MIF developer (Microchem) for $1 \mathrm{~min}$, and rinsed by DI water.

Magneto-electrical characterization was performed based on conventional 4-point method. The GMR sensor was placed in between pole shoes of the electromagnets, where uniform in plane magnetic field was cycled between $\pm 25 \mathrm{mT}$ and the sensor resistance was recorded. The GMR sensor is a proximity sensor, which responses to local magnetic stray fields from the objects. Therefore, a strategy is taken to enhance the magnetic stray fields to be detected. As the ferrofluid (EMG 700, Ferrotec) employed for present study is a suspension of magnetic colloidal nanoparticles in water, it does not retain remanence without applying external magnetic field. Thus, an external field should be applied to achieve a net magnetization of the ferrofluid. In addition, the external magnetic field can be simultaneously used to bias the sensor to the sensitive region. Based on the above considerations, a permanent magnet (AlNiCo 500, type A1560, IBSMagnet, length: $60 \mathrm{~mm}$, diameter: $15 \mathrm{~mm}$ ) was placed below the GMR sensor which was mounted on a movable stage. With adjustment of the position of the permanent magnet, the sensor was biased to the most sensitive region and the stray fields from the magnetized ferrofluid were sufficient to be detected by the GMR sensor. Further, the tubing was placed in close contact to the sensor surface to enhance the stray fields that can be detected. As the GMR sensor is the proximity sensor, the transition from the dynamic to saturation regime (Supplementary Fig. 1b) is related to the dimension of the GMR sensor but not entirely to the geometrical parameters of the tube. Saturation regime is observed when the sensor width (dimension of the sensor in the direction of the droplet travel) is equal to the length of the droplet. In our experiments, width of the sensor is $1 \mathrm{~mm}$. Therefore, transition to saturation is observed when the droplet length is also about $1 \mathrm{~mm}$ (Supplementary Fig. 1b). In this case, the critical volume of the droplet assuming its shape of cylinder with two half-spheres at both ends can be estimated by taking into account width of the sensor (W) and radius of the fluidic tube (R): $V_{C}=4 / 3 \pi R^{3}+P_{i} R^{2}(W-2 R)$. For the geometry of our experiment, $\mathrm{V}_{\mathrm{C}} \sim 110 \mathrm{nl}$.

The GMR sensor was soldered and connected with 3 other trimmers (Bourns, 3006P-1-102LFto realize a Wheatstone bridge geometry (Supplementary Fig. 5c). By balancing the bridge, the background voltage from the bridge can be minimized to enhance the measuring sensitivity. The lock-in amplifier (SRS830) was used to amplify the signal and reduce the noise. The whole bridge circuit was powered also by lock-in with constant ac voltage of $0.800 \mathrm{~V}$. The modulation frequency was $1 \mathrm{kHz}$ and the time constant was $10 \mathrm{~ms}$. The sensitivity of lock-in was set at $500 \mu \mathrm{V}$ with a resolution of $10 \mathrm{nV}$, which was sufficient to resolve the voltage signal. The analog output from the lock-in was sampled at $512 \mathrm{~Hz}$ and transferred to the computer. Affiliated to the sensing platform a digital camera (DNT Microdigit) was installed which was used to monitor the flowing droplets. Videos and pictures of flowing droplets can be correlated with the voltage signals obtained with the GMR sensor.

GMR-based sorting module. The droplet generation module was refined to form a train of droplets of multiple sizes for the sorting (Supplementary Fig. 5a). The module was composed of two $\mathrm{T}$-junctions (C1and $\mathrm{C} 2$ ) with different thru-hole diameters (C1: $0.5 \mathrm{~mm}, \mathrm{C} 2: 0.25 \mathrm{~mm})$. Due to the differences in the size of thru-hole diameter, droplets of distinct size can be produced at these two junctions. Big droplets were formed in the junction $\mathrm{Cl}$ using syringe $\mathrm{A}$, while smaller droplets were injected into the same fluidic channel through the junction $\mathrm{C} 2$ through syringe $\mathrm{C}$. Such fluidic assembly resulted in the sequence of the droplets with alternating size, which was further used for sorting experiments. The sorting part was composed of a T-junction (Supplementary Fig. 5d, thru-hole diameter: $0.25 \mathrm{~mm}$ ) flanked by two separate fluidic channels that were connected to a solenoid 3-way isolation valve (Amico Scientific) as mentioned before. The 3-way valve was with one-way open and one-way closed without supplying power. The switching of the valve was controlled by an external power source (Agilent Precision Source/Measurement Units B2902A) with working voltage of $24 \mathrm{~V}$. In the sorting experiment, applied voltage of $20 \mathrm{~V}$ with 
compliance of $0.5 \mathrm{~mA}$ was sufficient to switch on the valve. The algorithm of magnetic sorting is described as follows: ferrofluid droplets of different size are detected by the GMR-based millifluidic droplet detector. The electrical signal from the detector is analyzed and compared to the threshold value. A threshold voltage is defined to trigger the on/off performance of the solenoid 3-way isolation valve installed behind the sensor, so as to allow the droplets of interest to be sorted into the corresponding channel. A hold-time and delay-time are set to determine how long the valve to be kept open and when the opening of the valve will be delayed after receiving a signal trigger from the GMR sensor. This feature is crucial when the spacing between droplets is smaller than the distance between the GMR sensor and the sorting junction.

Multiparametric analysis. The detection events of ferrofluid droplets are represented by the detected peaks (Supplementary Fig. 1a). Each peak can be characterized by its amplitude, the full-width at half maximum (FWHM), positive/negative amplitude ratio (PNR). These characteristics can serve as fingerprints for each ferrofluid droplet. For instance, the amplitude is related to the size and concentration of droplets, the FWHM contains the information about the speed and size of droplets, while the orientation of magnetization of the ferrofluid droplets is relevant to the PNR. These distinct features can be used to discriminate each labeled magnetic object. We performed the following experiments at a fixed flow conditions. Therefore, modifications of the FWHM are mainly attributed to the different size of droplets. Based on the previous studies, the size and concentration of ferrofluid droplets can be controlled and deterministically correlated to the derived voltage change.

We performed multiparametric analysis on the ferrofluid emulsion droplets. The amplitude and FWHM of the detection peak were derived by a Gaussian fit to the peak and taken for the established parametric analysis. Since the amplitude and FWHM are related to the droplet size and flowing speed, the distribution of the droplet size and amount of ferrofluids under fixed flow condition can be evaluated. FWHM is dependent not only on the size of droplets but also on their velocities. Therefore, the combined multiparametric analysis of the amplitude and FWHM can be used for the evaluation of the droplet size as well as the flow conditions. The multiparametric density plots for the ferrofluid droplets (with concentration of $15 \mathrm{mg} / \mathrm{ml}$ ) with different volumes are summarized in Figure 2a (in the manuscript). The plots collect over about 100 droplets. The broad distribution of the FWHM and amplitude (black cloud, Fig. 2a, in the manuscript) is consistent with the real time detection results (Supplementary Fig. 1a). Also the corresponding fitted histogram displayed in Figure $2 c$ (in the manuscript) indicates a clear polydispersity of droplet size. The droplet volume increases with the increase of flow rate of ferrofluids. The size of ferrofluid droplets is smaller than $1 \mathrm{~mm}$ for the flow rate of ferrofluid up to $200 \mathrm{nl} / \mathrm{s}$. Thus the sensor is still working within the dynamic range as marked by the blue region in Supplementary Figure 1b. The amplitude of voltage change is sensitive to the change of droplet size. Thus, a narrow distribution of the data point cloud and the decrease standard deviation of the amplitude and FWHM in the fitted histograms (Fig 2c, in the manuscript) indicates a more uniform distribution of droplet size at larger volumes. The standard deviations are derived by a Gaussian fit to the histograms of amplitude and FWHM, respectively. The elliptical circles in Figure 2a,b in the manuscript are guide to the eyes. The elliptical circle is located at the center of the Gaussian fits to the histograms of the amplitude and FWHM, while the long and short axis of the elliptical circles take the half width values of the Gaussian fit to the amplitude and FWHM histograms. With droplet volume of $270 \mathrm{nl}$, the sensor is saturated. The variation of voltage change amplitude can reflect the concentration variation. However, in this case, the analysis shows that the standard deviation of amplitude is below $2 \mu \mathrm{V}$ (see Supplementary Fig. 2i), which is smaller than the binning size of the histogram and close to the noise level (around $1.5 \mu \mathrm{V}$ ). This indicates that concentration variation per droplet contributes to the change of amplitude to lesser extent in the experiments. The histogram of amplitude can clearly reflect the size distribution of droplets when the sensor is still working in the dynamic region. However, as mentioned, when the size of droplets is larger than the sensor width, the sensor response is saturated with even larger size of droplets. Nonetheless, from the FWHM, the information of droplet size can still be extracted, which can be seen from the fact that the magnitude of FWHM increases as the droplet volume increases from $120 \mathrm{nl}$ to $270 \mathrm{nl}$.We next performed the multiparametric analysis of droplets with different concentrations which were produced under the same flow condition $\left(\mathrm{Q}_{\mathrm{oil}}=500 \mathrm{nl} / \mathrm{s}, \mathrm{Q}_{\mathrm{ferro}}=300 \mathrm{nl} / \mathrm{s}\right)$. The clouds of detection characteristics for droplets with different concentrations naturally fall into the same FWHM region (Supplementary Fig. 2e-h) indicating the consistent, homogeneous distribution of the droplet size for each specified concentration. The standard deviation of FWHM and amplitude for each specified concentrations levels off (Supplementary Fig. 2j), which indicates a relative consistent and homogenous droplets produced under the same flow conditions for each specified concentration. However, due to the scaling of voltage change with the magnetic moment, the center of the data point cloud is clearly shifted to higher voltage amplitudes for droplets with larger concentration of magnetic nanoparticles.

1. de Bono, J. S. \& Ashworth, A. Translating cancer research into targeted therapeutics. Nature 467, 543-9 (2010).

2. Tseng, P., Judy, J. W. \& Di Carlo, D. Magnetic nanoparticle-mediated massively parallel mechanical modulation of single-cell behavior. Nature methods 9, 1113-9 (2012).

3. Sabaté, R., Barnadas-Rodríguez, R., Callejas-Fernández, J., Hidalgo-Alvarez, R. \& Estelrich, J. Preparation and characterization of extruded magnetoliposomes. International journal of pharmaceutics 347, 156-62 (2008).

4. Alexiou, C. et al. In vitro and in vivo investigations of targeted chemotherapy with magnetic nanoparticles. Journal of Magnetism and Magnetic Materials 293, 389-393 (2005).

5. Doerr, A. Multiplexing MRI. Nature Methods 5, 668-668 (2008).

6. Kourilov, V. \& Steinitz, M. Magnetic-bead enzyme-linked immunosorbent assay verifies adsorption of ligand and epitope accessibility. Analytical Biochemistry 311, 166-170 (2002).

7. Mönch, I. et al. Rolled-up magnetic sensor: nanomembrane architecture for inflow detection of magnetic objects. ACS nano 5, 7436-42 (2011).

8. Shen, W., Liu, X., Mazumdar, D. \& Xiao, G. In situ detection of single micronsized magnetic beads using magnetic tunnel junction sensors. Applied Physics Letters 86, 253901 (2005).

9. Krutzik, P. O. \& Nolan, G. P. Fluorescent cell barcoding in flow cytometry allows high-throughput drug screening and signaling profiling. Nature methods 3, 361-8 (2006).

10. Kiermer, V. FACS-on-a-chip. Nature Methods 2, 91-91 (2005).

11. Boedicker, J. Q., Li, L., Kline, T. R. \& Ismagilov, R. F. Detecting bacteria and determining their susceptibility to antibiotics by stochastic confinement in nanoliter droplets using plug-based microfluidics. Lab on a chip 8, 1265-72 (2008).

12. Baraban, L. et al. Millifluidic droplet analyser for microbiology. Lab on a chip 11, 4057-62 (2011)

\section{Acknowledgments}

The authors thank I. Fiering (IFW Dresden) for sample deposition and M. Melzer (IFW Dresden) for valuable discussions. This work is financially supported by the DFG Research Group 1713 and European Research Council under the European Union's Seventh Framework Programme (FP7/2007-2013)/ERC grant agreement n ${ }^{\circ}$ 306277. Furthermore, we gratefully acknowledge support from the German Excellence Initiative via the Cluster of Excellence EXC 1056 Center for Advancing Electronics Dresden (cfAED).

\section{Author contributions}

L.B., D.M., G.C., O.G.S. wrote the proposals and oversaw the research in their groups. L.H. and D.K. assisted with sample characterization and experiments. G.L. and L.B. fabricated the samples. G.L. and L.H. conceived and performed the experiments, conducted data analysis and carried out simulations. G.L., D.M., L.B., O.G.S. wrote the paper. All authors co-wrote the paper and agree to its contents.

\section{Additional information}

Supplementary information accompanies this paper at http://www.nature.com/ scientificreports

Competing financial interests: The authors declare no competing financial interests. How to cite this article: Lin, G.G. et al. Magnetoresistive Emulsion Analyzer. Sci. Rep. 3, 2548; DOI:10.1038/srep02548 (2013).

This work is licensed under a Creative Commons AttributionNonCommercial-NoDerivs 3.0 Unported license. To view a copy of this license, visit http://creativecommons.org/licenses/by-nc-nd/3.0 